\title{
Uma reflexáo sobre as parasitoses intestinais em comunidades de baixa renda do norte do Estado do Rio de Janeiro, Brasil.
}

\section{A reflection on the intestinal parasitic infections in low-income communities from north of Rio de Janeiro State, Brazil.}

\author{
Antonio Henrique Almeida de Moraes Neto ${ }^{1}$ \\ Clóvis de Paula Santos ${ }^{2}$ \\ João Carlos de Aquino Almeida ${ }^{2}$
}

\section{Resumo}

No presente trabalho são discutidos os fatores que influenciam a transmissão e a prevalência de parasitoses intestinais em comunidades de baixa renda do norte do Estado do Rio de Janeiro, levando-se em conta os resultados obtidos de 2004 a 2009 no âmbito do Programa Parasitoses do Norte Fluminense, desenvolvido em parceria entre a FIOCRUZ, UENF e diversas instituiçôes de pesquisa e ensino superior do Rio de Janeiro. São abordadas sugestôes para a redução da prevalência nestas comunidades, tomando-se por base o Plano Nacional de Vigilância e Controle das Enteroparasitoses, o Programa Saúde na Escola do Governo Federal, o Plano Nacional de Saúde Ambiental e a Resolução 54.19 de 2001 da Assembléia Mundial da Saúde, Organização Mundial da Saúde e as metas de desenvolvimento sustentável do Milênio.

Palavras-chave: Parasitoses intestinais, comunidades carentes, educação, saúde.

\section{Abstract}

In this paper we discuss the factors that influence the prevalence and transmission of intestinal parasitic infections in low-income communities in northern Rio de Janeiro, taking into account the results obtained from 2004 to 2009 in the framework of the Parasites of North Fluminense, developed in partnership between the FIOCRUZ, UENF and other institutions of research and higher education in Rio de Janeiro. It covers suggestions for reducing the prevalence in these communities, taking as basis the National Monitoring and Control of Intestinal Parasites, the School Health Program of the Federal Government, the National Environmental Health and Resolution 54.19 of 2001 from the World Health Organization and the goals of milenium sustainable development.

Keywords: Instestinal parasitic infections, low-income communities, education, health. 


\section{INTRODUÇÃO}

O Plano Nacional de Saúde Ambiental (PNSA), estabelece que Saúde Ambiental é a área da Saúde Pública que abrange o conhecimento cientifico e a formula $\neg$ ção de políticas públicas relacionadas à interação entre a saúde humana e os fatores do meio ambiente natural e antrópico que a determinam, condicionam e influenciam, com vistas a melhorar a qualidade de vida do ser humano, sob o ponto de vista da sustentabilidade (Tavares et al 2001).

De acordo com a Lei Federal 11.445 de 2007 os municípios brasileiros são obrigados a desenvolverem um plano de saneamento básico até 2010, mas muitas cidades permanecem sem condiçóes de colocá-lo em prática. Segundo dados da OMS, para cada $\mathrm{R} \$ 1,00$ investido em saneamento se economiza $\mathrm{R} \$$ 4,00 com saúde pública. Cerca de $80 \%$ das doenças conhecidas são relacionadas à água contaminada e $70 \%$ da população rural e $25 \%$ da população urbana dos países em desenvolvimento não dispóem de água potável. No mundo, estima-se que ocorram cerca de 900 milhôes de casos de diarréia e dois milhóes de óbitos infantis por ano, devido ao consumo de água contaminada. As doenças de veiculação hídrica mais comuns são: a febre tifóide, disenteria, cólera, diarréia, hepatite, leptospirose e giardíase entre outras parasitoses intestinais (http://www. who.int). No Brasil, de acordo com estimativas do Ministério da Saúde, a metade das mortes de crianças até um ano de idade e das internaçóes hospitalares estão associadas a doenças transmitidas pela água (Zancul, 2006).

As parasitoses intestinais representam um importante problema de saúde pública em paises em desenvolvimento (WHO, 2005). No Brasil, existem poucos dados fidedignos sobre a prevalência destas parasitoses, pois baseam-se em estimativas e na maioria dos casos ocorre a subnotificação por parte dos serviços de atenção básica a saúde, levando a índices dúbios, em áreas onde predominam condiçóes precárias de saneamento básico e moradia, bem como dificuldade de acesso a atendimento médico e a informaçóes sobre profilaxia e prevenção (Bencke et al. 2006). Além disso, somam-se a estes fatores as características regionais de cada comunidade, onde se sobressaem crenças enraizadas que são perpetuadas entre geraçóes e que só tornam o quadro ainda mais complicado (Mello et al 1988).

Neste contexto, recentemente realizamos um levantamento sobre parasitoses intestinais no norte do Estado do Rio de Janeiro, no âmbito do Programa Parasitoses do no Norte Fluminense, realizado em parceria entre diversas Instituiçôes de Ensino e Pesquisa na área de Saúde Pública no Estado do Rio de Janeiro com o apoio da Fundação Carlos Chagas Filho de Amparo a pesquisa do Estado do Rio de Janeiro, FAPERJ, onde observamos que a maioria dos mo- radores, professores da rede pública e privada, técnicos de nível médio da área de saúde pública, estudantes de nível superior da área biomédica do município de Campos dos Goytacazes e em especial residentes e escolares das comunidades de Ururaí, Travessão e Assentamento Oziel Alves (Movimento Sem Terra - MST), Campos dos Goytacazes, $\mathrm{RJ}$, apresentaram pouco ou nenhum conhecimento sobre a transmissão e prevenção das parasitoses intestinais. Tal fato, no caso dos residentes e escolares das comunidades carentes de Ururaí, Travessão e Assentamento Oziel Alves, estava associado à baixa renda (em torno de 1 salário mínimo), escolaridade (nível fundamental incompleto) e péssimas condiçôes de saneamento básico nestas localidades. Estes indivíduos acreditam que: "se pega verminose comendo doce, bebendo leite ou café", que não se deve tomar remédio contra verminoses no mês de agosto, pois é o mês da revolta dos vermes" e que "se deve tomar remédio para prevenir as verminoses". A taxa de prevalência de parasitoses intestinais nas famílias residentes nestas comunidades foi $28,3 \%$ ( $n=$ 550 famílias), enquanto que na população escolar na faixa de 10 a 18 anos foi de $37,1 \%(n=2.000$ escolares $)$. O parasita mais frequente foi Giardia lamblia, que é transmitido por veiculação hídrica refletindo as precárias condiçôes de saneamento básico nas comunidades estudadas Paralelamente a este estudo desenvolvemos pesquisa nutricional, onde tecnicamente apesar de não termos observado correlação entre nutrição e prevalência de parasitoses intestinais, constatamos que a ingesta alimentar das famílias residentes nas comunidades carentes era inadequada, devido ao baixo consumo de nutrientes essenciais (frutas e legumes) e a excessiva ingestão de carboidratos, o que pode ocasionar diversas patologias relacionadas (Moraes Neto et al 2009 A e B, no prelo). Por outro lado, de acordo com Ferreira et al. 2000, citado no Plano Nacional de Vigilância e Controle das Enteroparasitoses (PNVCE) (SVS, 2005): "cabe ressaltar que em nenhum inquérito envolvendo saúde e nutriçáo no Brasil, foi realizado o exame parasitológico de fezes"; o que remete a importância de estudos em epidemiologia de parasitoses intestinais, o que não vem sendo preconizado e priorizado pelas agências de fomento.

No caso dos professores e mesmo aqueles profissionais e estudantes de nível médio e superior ligados a área de Saúde Pública (inclusive técnicos de nível médio em Patologia Clínica), constatamos, através de testes realizados sobre o conhecimento destes sobre o assunto, durante um curso de atualização em saúde ministrado e direcionado a este segmento, ao longo de três anos (2007 a 2009) em Campos dos Goytacazes, RJ, que o quadro é ainda mais grave, pois além de manifestarem aquelas crendices já citadas pela população em geral, apresentavam também enorme defasagem de aprendizagem e conteúdo relacionado a transmissão e prevençâo das 
parasitoses intestinais. No caso das autoridades municipais, apesar de apoiar o projeto em causa, pouco esforço e interesse foi manifestado durante os anos do estudo (2004 a 2009) para a efetiva mudança das condiçóes de saneamento básico na área afim de se obter redução nos índices de prevalência e ganho de conhecimento por parte dos moradores, nos indicadores educacionais relacionados ao tema.

De acordo com o PNSA, o Sistema Único de Saúde (SUS), deve promover as adequaçóes necessárias para a melhoria da atenção básica a saúde, no que diz respeito a assistência médica especializada, previlegiando a prevenção e o controle de doenças e a promoção da saúde. Pouco tem sido feito nesta esfera da administração da saúde pública, principalmente no que diz respeito as comunidades carentes, onde a transmissão das parasitoses intestinais assumem características alarmantes.

Estes dados refletem incongruências no PNVCE, no âmbito da Secretaría de Vigilância Sanitária (Ministério da Saúde), onde os índices nacionais e regionais permanecem baseados em estimativas.

O PNVCE (SVS, 2005) estabelece que "esteja nele baseada a utilização de informaçóes sobre prevalência, morbidade e mortalidade causadas ou associadas às infecçôes por parasitas, gerando estudos analíticos desenhados a partir da avaliação epidemiológica dos dados registrados, e visa à definição de estratégias para o controle das enteroparasitoses. Deverá ser instituído e constituído, respeitadas as áreas de competência e níveis governamentais, pelos órgãos que desenvolvem atividades de Vigilância Epidemiológica, Sanitária e Ambiental, Saneamento, Educação em Saúde, Diagnóstico e Assistência”. O que se denota é uma assincronia entre os níveis governamentais e total desconhecimento dos índices reais de prevalência e morbidade por parte dos gestores assim como da população como um todo, em comunidades de baixa renda, das medidas de prevenção e controle.

Por outro lado, há a necessidade premente do ensino de Parasitologia ser revisto nas carreiras ligadas a área médica, nas Instituiçôes de Ensino Superior (IES). Os conteúdos são compartimentalizados e os conceitos deturpados pelos alunos. Os professores necessitam de reciclagem e existe a excessiva prática de aulas teóricas em detrimento das práticas, o que atenua a baixa qualidade do processo ensino-aprendizagem. Apesar de tentativas de integração entre o ensino técnico e a pesquisa científica terem sido desenvolvidas por estudos acadêmicos (Velozo et al 1999; Minayo 1992; Paoli 1988), tal discrepância se reflete no ensino de nível fundamental onde as crianças em idade escolar acreditam que "os vermes são terríveis vírus e bactérias” e que "a água boa para consumo é aquela transparente”. Práticas educativas inovadoras levando a ciência e arte para a sala de aula tem trazido resultados animadores, a partir da participação dos alunos e engajamento de professores e gestores de ensino e da escola como um todo, para a prevenção de doenças (Japiassú, 1998). No entanto, as ações de prevenção para as parasitoses intestinais deveriam ser melhor exploradas e estimuladas no ambiente escolar, uma vez que o Programa Saúde na Escola (PSE) do Governo Federal, instituído pelo decreto presidencial no 6.286, de 5 de dezembro de 2007, prevê uma política intersetorial entre os Ministérios da Saúde e da Educação na perspectiva da atenção integral (prevenção, promoção e atenção) à saúde de crianças, adolescentes e jovens do ensino básico público (educação infantil, ensino fundamental e médio, educação profissional e tecnológica e na educação de jovens e adultos (EJA), no âmbito das escolas e/ou das unidades básicas de saúde, realizadas pelas Equipes de Saúde da Família (Programa Saúde da Família - PSF). O sistema de ensino do Brasil tem mais de sete milhóes de crianças matriculadas na Educação Infantil (www.mec.gov.br), porém há municípios, onde o PSF sequer existe ou mesmo foi desativado e nestes não há política para o controle das enteroparasitoses para os grupos vulneráveis da população de baixa renda.

Desta forma, estamos longe de atender as demandas da Assembléia Mundial da Saúde que determina a seus países membros, inclusive o Brasil, através da resolução 54.19 de 2001, que $75 \%$ das crianças em idade escolar e populaçóes vulneráveis devem ser desverminadas até o ano de 2010, a fim de atingir as metas de desenvolvimento sustentável do milênio (WHO, 2005).

\section{REFERÊNCIAS BIBLIOGRÁFICAS}

Bencke A, Artuso GL, Reis RS, Barbieri NL, Rott MB (2006) Enteroparasitoses em escolares residentes na periferia de Porto Alegre, RS, Brasil. Rev Pat Trop (35): 31-36

Duarte EC, Schneider MC, Paes-Sousa R, Ramalho WM, Sardinha LMV, Silva Júnior JB, Castillo-Salgado C (2002) Epidemiologia das desigualdades em saúde no Brasil: um estudo exploratório. Fundação Nacional de Saúde, Centro Nacional de Epidemiologia, Cenepi - Brasília, Brasil, Organização Pan-Americana de Saúde, 123 pp

Ferreira UM, Ferreira CS, Monteiro CA (2000) Tendência secular das parasitoses intestinais na infância na cidade de São Paulo (1984-1996). Rev Saúde Pública, 34:73-82

Japiassu ROV (1988) Jogos teatrais na escola pública. Rev Fac Educ (24): 81-97

Mello DA, Pripas S, Fucci M, Santoro MC, Pedrazani ES (1988) Helmintoses intestinais: conhecimentos, atitudes e percepção da população. Rev Saúde Publ 22: 140-149 
Minayo, MCS (1992). O desafio do conhecimento: a pesquisa qualitativa em saúde. São Paulo: Editora Hucitec/Rio de Janeiro: Abrasco.

Moraes Neto AHA, Pereira APMF, Alencar MFL, Almeida MCR, Souza-Júnior PRB, Dias RC, Fonseca JG, Mattias L, Santos CP, Almeida JCA (2009 a) Prevalence of intestinal parasites versus knowledge and perceptions of inhabitants of low-income communities of Campos dos Goytacazes, Rio de Janeiro State, Brazil. Acta Tropica, in press

Pereira APMF, Almeida MCR, Alencar MFL, Souza-Júnior PRB, Dias RC, Cecchetto F, Dias RC, Aguiar MF, Santos CP, Almeida JCA, Moraes Neto AHA (2009 b) The influence of health education to reduce the prevalence of intestinal parasitic infections in low-income communities from North of Rio de Janeiro State, Brazil. Mem Inst Oswaldo Cruz, in press

Paoli N (1988). O princípio da indissociabilidade do ensino e da pesquisa: elementos para uma discussão. Cad Cedes, 22: $27-52$

SVS (2005) Plano nacional de vigilância e controle das enteroparasitoses. Secretaria de Vigilância em Saúde, Ministério da Saúde, Brasília, DF, Brasil. 44 pp

Tavares MSOC, Franco-Netto G, Aragão LGT, Carneiro FF, Câncio J (2001) Construindo a política nacional de saúde ambiental. Ministério da Saúde, Brasil

Velozzo V, Martins MIC, Nascimento RB (1999) Articulando ensino e pesquisa:

construindo uma proposta de capacitação para docentes do ensino técnico Cad Saúde Pública 15 (Sup. 2): 29-37

WHO (2005) Deworming for health and development. Report of the third global meeting of the partners for parasite control. World Heath Organization. 51 pp

Zancul MS (2006). Água e saúde. Rev Eletr Ciências (32): $1-3$

http://www.mec.gov.br , acessado em 13/09/2009

Endereço para Correspondência:

http://www.who.int, acessado em 08/09/2009

Antonio Henrique Almeida de Moraes Neto ahmn@ioc.fiocruz.br ou ahmn.ioc@gmail.com FIOCRUZ, Instituto Oswaldo Cruz - IOC, Laboratório de Ecoepidemiologia e Controle da Esquistossomose e Geohelmintoses - LECEG Pavilhão Lauro Travassos, $2^{\circ}$ Andar Avenida Brasil 4365 